\title{
Modelling and Analysis of Retinal Ganglion Cells Through System Identification
}

Kerr, D., McGinnity, M., \& Coleman, SA. (2014). Modelling and Analysis of Retinal Ganglion Cells Through System Identification. In Unknown Host Publication (pp. 158-164). SciTePress.

https://doi.org/10.5220/0005069701580164

Link to publication record in Ulster University Research Portal

\section{Published in:}

Unknown Host Publication

Publication Status:

Published (in print/issue): 01/01/2014

\section{DOI:}

10.5220/0005069701580164

\section{Document Version}

Publisher's PDF, also known as Version of record

\section{General rights}

Copyright for the publications made accessible via Ulster University's Research Portal is retained by the author(s) and / or other copyright owners and it is a condition of accessing these publications that users recognise and abide by the legal requirements associated with these rights.

\section{Take down policy}

The Research Portal is Ulster University's institutional repository that provides access to Ulster's research outputs. Every effort has been made to ensure that content in the Research Portal does not infringe any person's rights, or applicable UK laws. If you discover content in the Research Portal that you believe breaches copyright or violates any law, please contact pure-support@ulster.ac.uk. 


\title{
Modelling and Analysis of Retinal Ganglion Cells Through System Identification
}

\author{
Dermot Kerr ${ }^{1}$, Martin McGinnity ${ }^{2}$ and Sonya Coleman ${ }^{1}$ \\ ${ }^{1}$ School of Computing and Intelligent Systems, University of Ulster, Magee campus, Derry, Northern Ireland \\ ${ }^{2}$ School of Science \& Technology, Nottingham Trent University, Nottingham, U.K.
}

Keywords: $\quad$ System Identification, Retinal Ganglion Cells, Linear-Nonlinear Model.

Abstract: $\quad$ Modelling biological systems is difficult due to insufficient knowledge about the internal components and organisation, and the complexity of the interactions within the system. At cellular level existing computational models of visual neurons can be derived by quantitatively fitting particular sets of physiological data using an input-output analysis where a known input is given to the system and its output is recorded. These models need to capture the full spatio-temporal description of neuron behaviour under natural viewing conditions. At a computational level we aspire to take advantage of state-of-the-art techniques to accurately model non-standard types of retinal ganglion cells. Using system identification techniques to express the biological input-output coupling mathematically, and computational modelling techniques to model highly complex neuronal structures, we will "identify" ganglion cell behaviour with visual scenes, and represent the mapping between perception and response automatically.

\section{INTRODUCTION}

Modelling biological systems is difficult due to insufficient knowledge about the internal components and organisation, and the complexity of the interactions within the system. System identification has emerged as a viable alternative to classical hypothesis testing for the understanding of biological systems and was first used to understand the responses of auditory neurons (De Boer, 1968). Using white noise stimuli as input, the output responses were recorded and inferences made on mapping the stimulus to the response. White noise stimulation is often selected to model biological vision systems (Sakai, 1988, Chichilnisky, 2001) as it is mathematically simple to analyse. However, it is unlikely that white noise stimuli would test the full function of a neuron's behaviour (Talebi, 2012). Thus, any model developed with this stimulus could only be considered a subset of the biological model under certain conditions.

In the work by Marmarelis (Marmarelis, 1972), the Wiener theory of nonlinear system identification was applied to study the underlying operation of the three stage neuronal structures in the catfish retina.
Following from this work, the Volterra-Wiener method has been used extensively to model nonlinear biological systems (Victor, 1977, 1979, Marmarelis, 2004, Korenberg, 1996). However, computational effort increases geometrically with the kernel order and in interpretation of higher order kernels (Herikstad, 2011). Marmarelis and Zhao (Marmarelis, 1997) presented a way of overcoming these limitations by developing a perceptron type network with polynomial activation functions.

Block-structured (Giri, 2010) or modular models in the form of cascaded or parallel configurations have been used to overcome the limitations of Volterra-Wiener models. Cascade models may take various forms such as linear-nonlinear (Ostojic, 2011), nonlinear-linear, linear-nonlinear-linear, etc. In particular, linear-nonlinear models have been used to describe the processing in the retina (Pillow, 2005).

The generalised modular model proposed by Korenberg (Korenberg, 1991) employed parallel linear-nonlinear cascades generating spike outputs with a threshold-trigger function. To model specific neuron responses such as burstiness, refractoriness and gain control, Pillow (Pillow, 2008) amended the linear-nonlinear models with feedback terms. Correlated neuron activity was modelled through the 
use of coupling filters (Pillow, 2008) to couple multiple linear-nonlinear models of individual cells.

Parametric system identification modelling techniques also exist. The NARMAX (nonlinear auto-regressive moving average with exogenous inputs) model (Billings, 1984) has been used to model the nonlinear behaviour observed in the fly photoreceptors (Friederich, 2009, Song, 2009). The NARMAX modelling technique is suitable for application in a number of areas and has also been used to model robot behaviour (Kerr, 2010), iceberg calving and detecting and tracking time-varying causality for EEG data (Billings, 2013). Neural network approaches have also been used to model biological aspects of the vision system. For example Lau (Lau, 2002) used a two layer neural network with the backpropagation training algorithm to model the nonlinear responses of neurons in the visual cortex to visual stimuli. Similarly, Prenger (Prenger, 2004) used a multilayer feed-forward neural network to model the nonlinear stimulusresponse relationship in the primary visual cortex using natural images.

In this paper we formalise and standardise the model development process by using system identification techniques (NARMAX modelling) to express the biological input-output coupling mathematically. We have used a NARMAX approach to obtain the models we need because:

- The NARMAX model itself provides the executable code straight away,

- The model is analysable, and gives us valuable information regarding

- How the model achieves the task,

- Whether the model is stable or not,

- How the model will behave under certain operating conditions, and

- How sensitive the model is to certain inputs, i.e. how "important" certain input are.

In Section 2 we present the visual stimuli used in the neuronal recordings to obtain the physiological data and in Section 3 we present an overview of the NARMAX modelling approach. Experiments and results are presented in Section 4 with discussion in Section 5 .

\section{NEURONAL DATA}

Recordings were obtained from isolated mice retinas under full field stimulation using a Gaussian white noise sequence as illustrated in Figure 1.

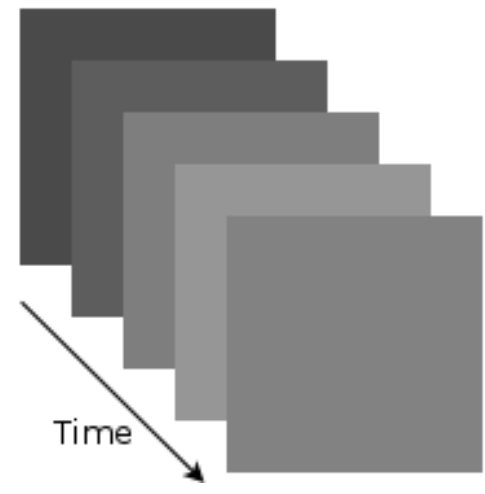

Figure 1: Full-field Gaussian white noise sequence.

The isolated retina was placed on a multi-electrode array, which recorded spike trains from many ganglion cells simultaneously. Stimuli were projected onto the isolated retina via a miniature cathode ray tube monitor. Spikes were sorted offline by a cluster analysis of their shapes, and spike times were measured relative to the beginning of stimulus presentation. In the experiments presented in this paper we analyse the response from an $\mathrm{ON}$ retinal ganglion cell (RGC).

\section{NARMAX MODELLING}

The NARMAX model is a difference equation that expresses the present value of the output as a nonlinear combination of previous values of the output, previous and/or present values of the input, and previous and/or present values of the noise signal. NARMAX is a parameter estimation methodology for identifying both the important model terms and the parameters of an unknown nonlinear dynamic system, such as a sensory neuron. For single-input single-output systems this model takes the form:

$$
\begin{array}{r}
y(k)=F[y(k-1), y(k-2), \ldots, y(k-n y), \\
u(k-d), \ldots, u(k-d-n u), \\
e(k-1), \ldots, e(k-n e)] \\
+e(k)
\end{array}
$$

where $y(k), u(k), e(k)$ are the sampled output, input and unobservable noise sequences respectively, $n y, n u, n e$ are the regression orders of $y(k), u(k), e(k)$ and $d$ is a time delay. $F[]$ is a nonlinear function and is typically taken to be a polynomial expansion of the arguments. Usually only the input and output measurements are 
available and the investigator must process these signals to estimate a model of the system.

The NARMAX methodology divides this problem into the following steps:

- Structure detection;

- Parameter estimation;

- Model validation;

- Prediction;

- Analysis.

These steps form an estimation toolkit that allows the user to build a concise mathematical description of the system (Billings and Chen, 1998). The procedure begins by determining the structure or the important model terms using a special orthogonal least squares procedure. This algorithm determines which dynamic and nonlinear terms should be in the model by computing the contribution that each potential model term makes to the system output. This allows the model to be built up term by term in a manner that exposes the significance of each new term that is added.

Structure detection is a fundamental part of the NARMAX procedure because searching for the structure ensures that the model is as simple as possible and a model with good generalisation properties is obtained. This approach mimics analytical modelling methods where the important model terms are introduced first. Subsequently the model is refined by adding in less significant effects. The only difference is that in the NARMAX method the model terms can be identified from the data set.

These procedures are now well established and have been used in many modelling domains. Once the structure of the model has been determined the unknown parameters in the model can be estimated. If correct parameter estimates are to be obtained the noise sequence, $e(k)$ which is almost always unobservable, must be estimated and accommodated within the model. Model validation methods are then applied to determine if the model is adequate.

Once the model has been determined to be adequate it can be used to predict the system output for different inputs. The model may also be used to study the characteristics of the system under investigation (Nehmzow, 2006). It is this latter aspect that is of particular interest in the work presented here. In this paper we have examined the suitability of NARMAX modelling to express the biological stimulus-response coupling mathematically and to validate the resulting stimulus-response couplings.

\section{MODEL IDENTIFICATION PROCEDURE AND ANALYSIS}

The proposed approach represents a decisive departure from current methods of generating retina models. We propose to "identify" (in the sense of system identification) the neuron's behaviour with natural visual scenes, and to represent the mapping between perception and response automatically, using the NARMAX system identification technique. We will illustrate how the various neural networks within the layered retina structure can be modelled using efficient polynomials that incorporate the neuron's nonlinear behaviour and dynamics. The compact polynomial representation will demonstrate that the intricate retina neural networks may be modelled in a compact compressed form.

\subsection{Data Pre-processing}

The overall goal of the pre-processing stage is to manipulate the data so that they form a regression dataset, i.e. input-output corresponding to the stimulus-response. In this case the dataset will be single-input single-output.

The Gaussian white noise is a stochastic highly interleaved stimuli spanning a wide range of visual inputs, is relatively robust to fluctuations in responsivity, avoids adaptation to strong or prolonged stimuli and is well suited to simultaneous measurements from multiple neurons. Examples of stimuli are presented in Figure 1 where each image in the sequence is presented sequentially to the isolated retina. As the stimulus has uniform intensity there is no need to extract the stimulus in the region of the receptive field.

Recordings of the ganglion cell neural response (spikes) to the full-field stimulation were supplied for two different ganglion cells in the case of this dataset. Each file contains the recorded times of spikes in seconds. For example,

[1.76304, 1.76912, 1.78504,..,546.63776].

Using these recorded spike times we compute a continuous temporal spike rate using the standard method of binning and convolution with a window function. Using this method we then have a continuous valued input-output dataset. For example, in Figure 2 we have illustrated the input data (stimulus intensity), recorded spikes and computed spike rate using an alpha window function for 500 milliseconds of a recording. Figure 2(a) illustrates the stimulus intensity, Figure 2(b) illustrates the recorded spikes, and Figure 2(c) 
illustrates the spike computed using a half wave rectified $\alpha$ function.

After this pre-processing stage we can use the system identification method to obtain a polynomial model that models the ganglion cells spike rate as a function of the stimulus intensity where the spatially uniform stimulus intensity is used as input (Figure 2(a)) and the computed spike rate (Figure 2(c)) is used as output.
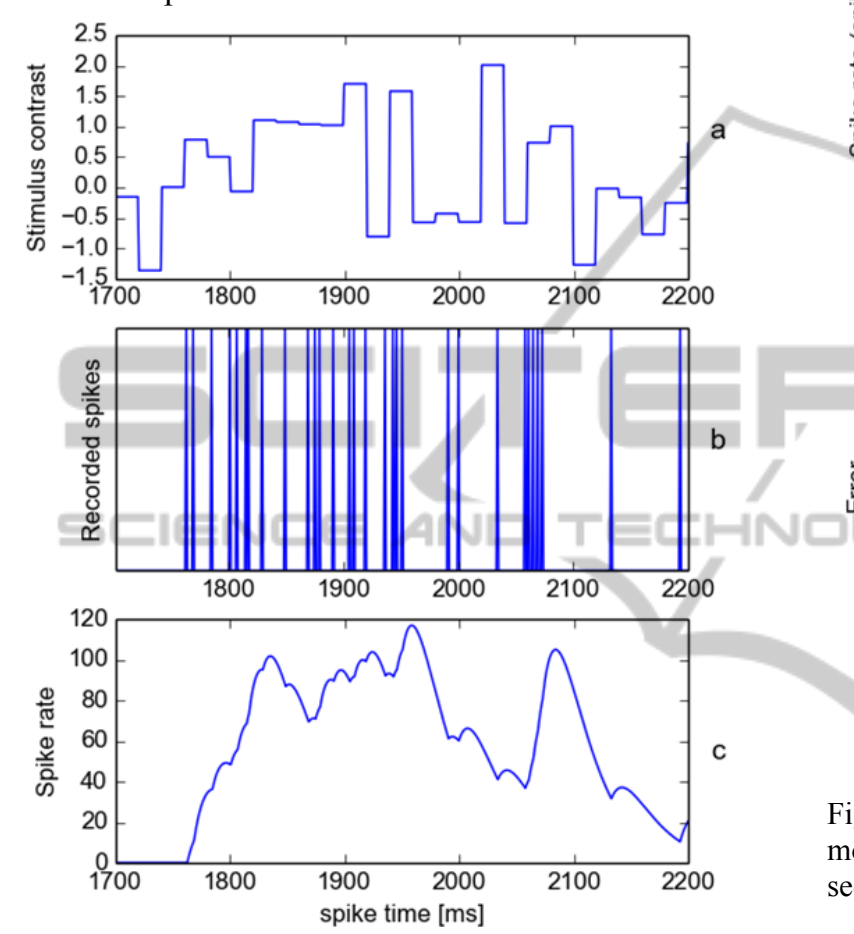

Figure 2: (a) Temporal stimulus intensity; (b) recorded spikes; (c) computed spike rate for $500 \mathrm{~ms}$ of a recording.

\subsection{Identifying the RGC Linear Model}

Using the NARMAX procedure outlined in Section 3 we construct a NARMAX model with an input regression of $n u=10$, corresponding to $200 \mathrm{~ms}$ of stimulus time and a polynomial of order 1 . The resulting model contained 11 terms and is presented in equation (2).

$$
\begin{aligned}
\operatorname{nr}(\mathrm{t})= & +10.8820504569 \\
& +0.4133355718 * \mathrm{u} 1(\mathrm{n}-1) \\
& +5.7712836251 * \mathrm{u} 1(\mathrm{n}-2) \\
& +11.1532223508 * \mathrm{u} 1(\mathrm{n}-3) \\
& +5.4095799493 * \mathrm{u} 1(\mathrm{n}-4) \\
& -0.9100060568 * \mathrm{u} 1(\mathrm{n}-5) \\
& -2.2967796022 * \mathrm{u} 1(\mathrm{n}-6) \\
& -1.5449416639 * \mathrm{u} 1(\mathrm{n}-7) \\
& -0.8126715957 * \mathrm{u} 1(\mathrm{n}-8) \\
& -1.1259153820 * \mathrm{u} 1(\mathrm{n}-9) \\
& -1.4256504406 * \mathrm{u} 1(\mathrm{n}-10)
\end{aligned}
$$

Using a new test stimulus sequence we then evaluate the performance of this NARMAX model and compare it to the actual neuronal response to the test stimulus. Results are presented in Figure 3.

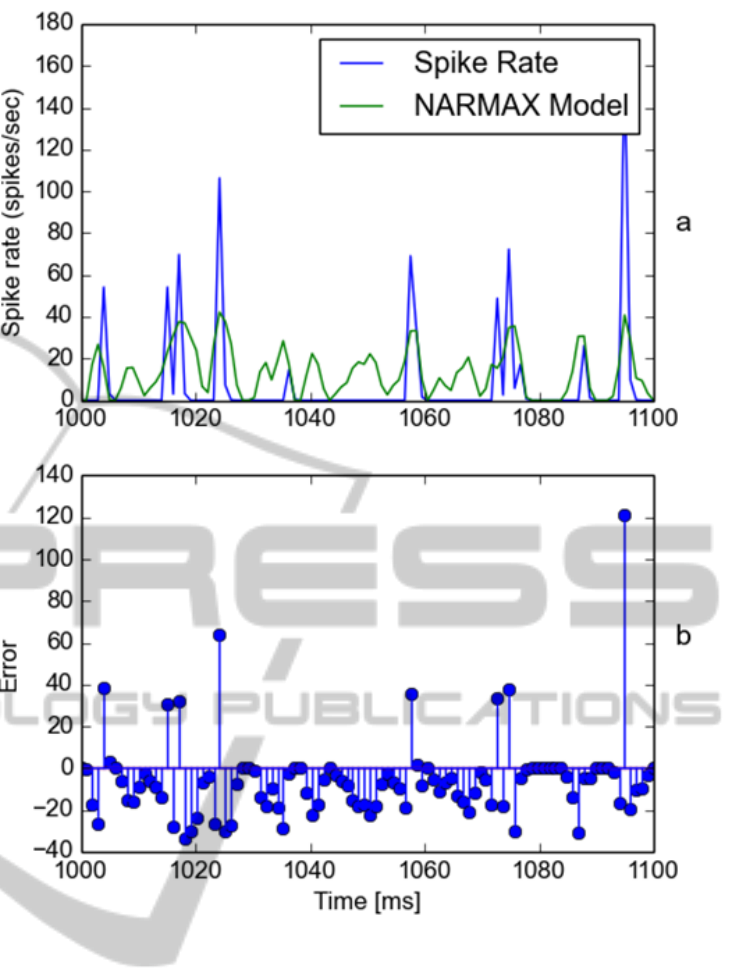

Figure 3: Comparison of linear polynomial NARMAX model and actual neuron response to novel test stimulus sequence.

Figure 3 (a) illustrates the actual recorded spike rate (blue) and the linear NARMAX model predicted spike rate (green). Figure 3 (b) illustrates the model error. We have also computed the RMSE as 20.92 and present a full comparison of RMSE results in Table 1.

\subsection{Identifying a RGC Quadratic Model}

Again using the same NARMAX procedure we construct a quadratic NARMAX model with an input regression of $n u=10$, corresponding to $200 \mathrm{~ms}$ of stimulus time and a polynomial of order 2 . The resulting quadratic polynomial model contained 26 terms and is presented in equation (3).

Figure 4 (a) illustrates the actual recorded spike rate (blue) and the quadratic NARMAX model predicted spike rate (green). Figure 4 (b) illustrates the model error. We have also computed the RMSE as 18.31 and present a full comparison of RMSE results in Table 1. Visual examination reveals both 
the linear and quadratic NARMAX models perform similarly with similar errors although the quadratic model has a reduced error compared to the linear model.

$$
\begin{aligned}
\mathrm{nr}(\mathrm{t})= & +5.96244435 \\
& +5.70629880 * \mathrm{u} 1(\mathrm{n}-2) \\
& +11.10949549 * \mathrm{u} 1(\mathrm{n}-3) \\
& +5.38644092 * \mathrm{u} 1(\mathrm{n}-4) \\
& -0.89662644 * \mathrm{u} 1(\mathrm{n}-5) \\
& -2.17791043 * \mathrm{u} 1(\mathrm{n}-6) \\
& -1.41390802 * \mathrm{u} 1(\mathrm{n}-7) \\
& -0.75777998 * \mathrm{u} 1(\mathrm{n}-8) \\
& -1.08523454 * \mathrm{u} 1(\mathrm{n}-9) \\
& -1.33837116 * \mathrm{u} 1(\mathrm{n}-10) \\
& +1.65057833 * \mathrm{u} 1(\mathrm{n}-2) \wedge 2 \\
& +4.28603998 * \mathrm{u} 1(\mathrm{n}-3) \wedge 2 \\
& -1.01260994 * \mathrm{u} 1(\mathrm{n}-5)^{\wedge} 2 \\
& +4.84809445 * \mathrm{u} 1(\mathrm{n}-2) * \mathrm{u} 1(\mathrm{n}-3) \\
& +1.55113103 * \mathrm{u} 1(\mathrm{n}-2) * \mathrm{u} 1(\mathrm{n}-4) \\
& -0.63963641 * \mathrm{u} 1(\mathrm{n}-2) * \mathrm{u} 1(\mathrm{n}-5) \\
& -0.71240927 * \mathrm{u} 1(\mathrm{n}-2) * \mathrm{u} 1(\mathrm{n}-6) \\
& +3.30083736 * \mathrm{u} 1(\mathrm{n}-3) * \mathrm{u} 1(\mathrm{n}-4) \\
& -1.14246640 * \mathrm{u} 1(\mathrm{n}-3) * \mathrm{u} 1(\mathrm{n}-5) \\
& -1.76540112 * \mathrm{u} 1(\mathrm{n}-3) * \mathrm{u} 1(\mathrm{n}-6) \\
& -0.95766543 * \mathrm{u} 1(\mathrm{n}-3) * \mathrm{u} 1(\mathrm{n}-7) \\
& -0.66097265 * \mathrm{u} 1(\mathrm{n}-3) * \mathrm{u} 1(\mathrm{n}-9) \\
& -1.10149068 * \mathrm{u} 1(\mathrm{n}-3) * \mathrm{u} 1(\mathrm{n}-10) \\
& -2.13889232 * \mathrm{u} 1(\mathrm{n}-4) * \mathrm{u} 1(\mathrm{n}-5) \\
& -1.68797033 * \mathrm{u} 1(\mathrm{n}-4) * \mathrm{u} 1(\mathrm{n}-6) \\
& -1.03141419 * \mathrm{u} 1(\mathrm{n}-5) * \mathrm{u} 1(\mathrm{n}-6) \\
&
\end{aligned}
$$

\subsection{Comparison with Linear-nonlinear Model}

To provide further comparison for the NARMAX models we evaluate against a standard benchmark by computing the Linear-Nonlinear (LNL) model (Ostojic, 2011). The first stage in computing the Linear-Nonlinear model is to compute the spike triggered average (STA) which is the average stimulus preceding a spike.

To compute the STA, the stimulus in the time window preceding each spike is extracted, and the resulting (spike-triggered) stimuli are averaged. Using the same dataset as the previous analysis we compute the STA and the results are presented in Figure 5. Here we can see that the RGC is an ON cell due to the positive peak in the temporal response of the filter. We can also see that the cell has a temporal memory of approximately $150-100 \mathrm{~ms}$. The plot illustrates the average values of stimulus intensity that elicit a response from the cell.

(3)
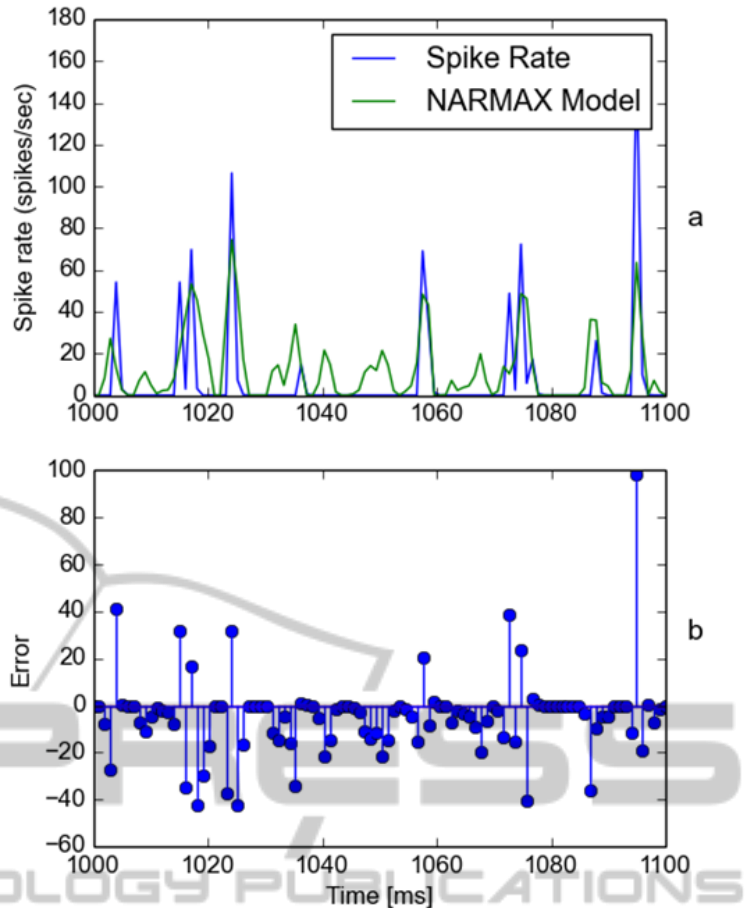

Figure 4: Comparison of quadratic polynomial NARMAX model and actual neuron response to novel test stimulus sequence.

The second stage in the Linear-Nonlinear model is used to re-construct the ganglion cells nonlinearity. We using the standard approach (Ostojic, 2011) of plotting the actual response against the STA predicted response, binning the values and fitting a curve using a cumulative density function. Figure 6 illustrates the obtained nonlinearity. Next, we apply the STA and nonlinearity to the same test stimulus and compute the response. We compare this estimated response to the actual neuronal response and plot the results as before. Results are presented in Figure 7.

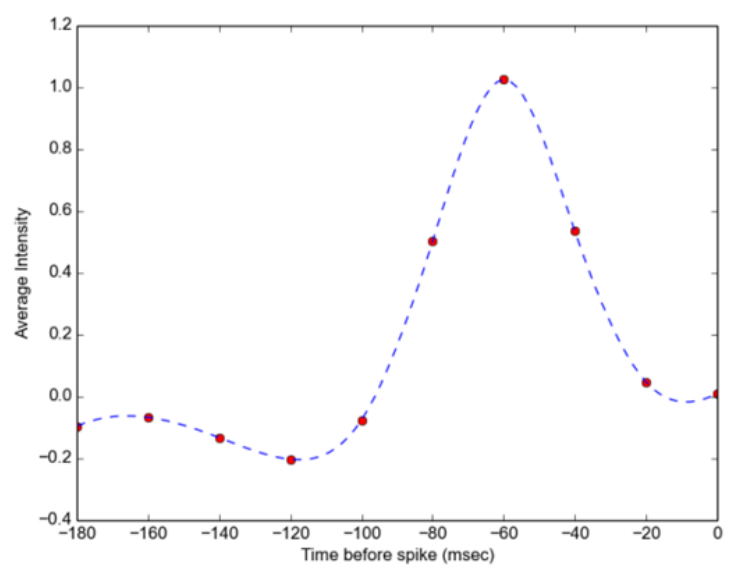

Figure 5: Computed spike triggered average for ON cell. 


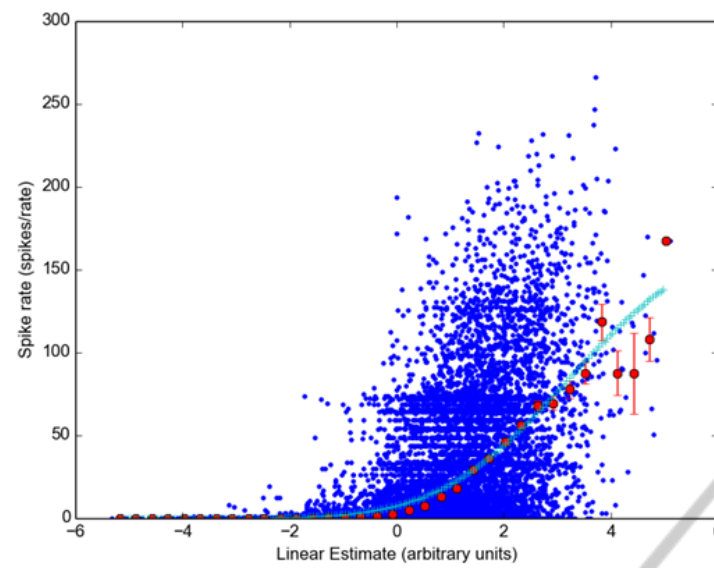

Figure 6: Nonlinearity computed for ON cell.

Figure 7 (a) illustrates the actual recorded spike rate (blue) and the LNL model predicted spike rate (green). Figure 7 (b) illustrates the model error. We have also computed the RMSE as 32.29 and present a full comparison of RMSE results in Table 1 . The RMSE results illustrate that the quadratic NARMAX model performs best. We have also plotted the actual neural response, LNL model predicted response and the quadratic NARMAX model predicted response in Figure 8 to illustrate comparative model accuracy. Visual examination reveals the quadratic NARMAX model has improved accuracy over the LNL model and is comparable to the actual neural response.
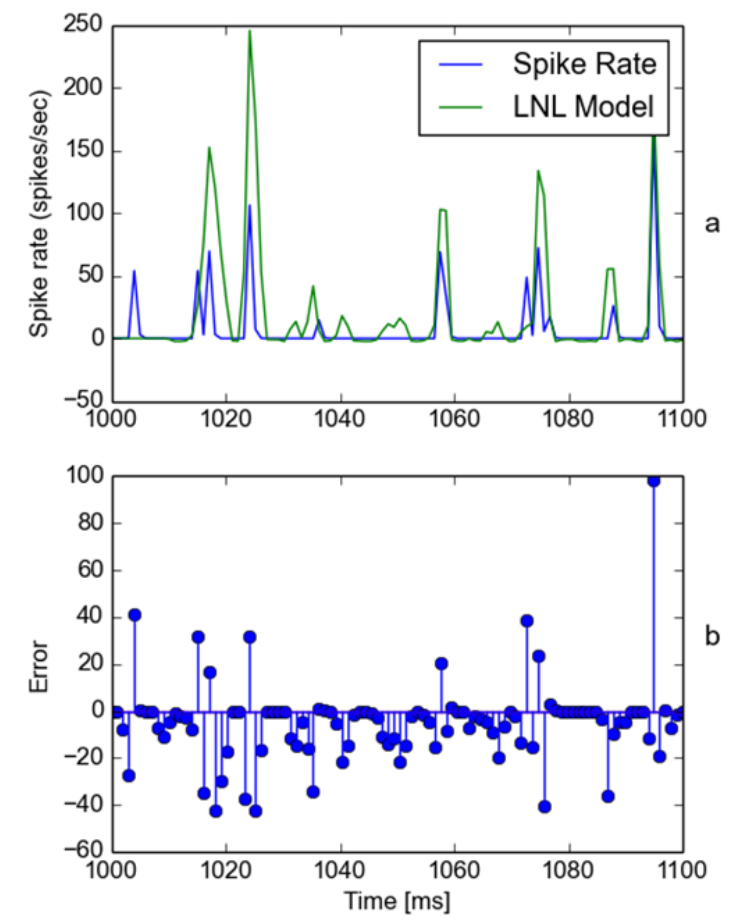

Figure 7: Comparison of Linear-Nonlinear model and actual neuron response to novel test stimulus sequence.

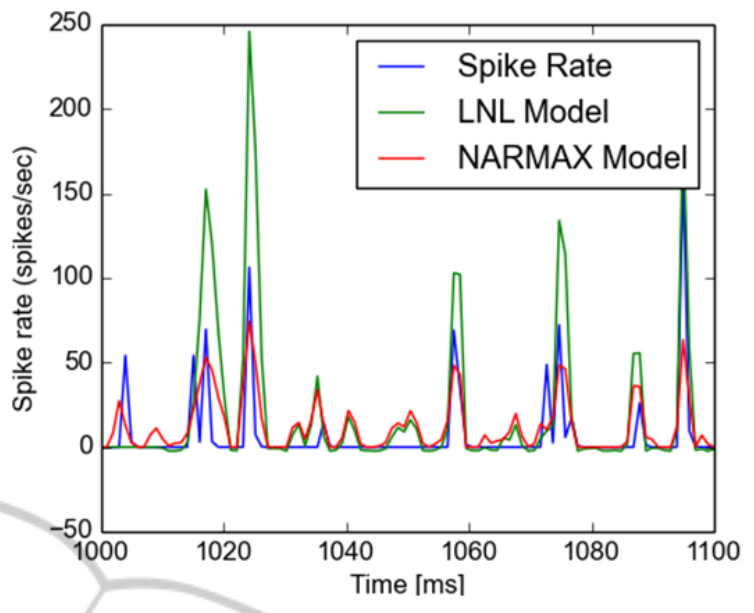

Figure 8: Comparative evaluation of actual spike rate, linear nonlinear model and quadratic NARMAX model.

Table 1: Summary of RMSE for linear NARMAX, quadratic NARMAX and Linear-Nonlinear models. The computed RMSE values illustrate that the quadratic NARMAX approach results in the best fitting model for the selected dataset. $\square \equiv L I C A T \| \square N \equiv$

\begin{tabular}{|l|c|}
\hline Method & RMSE \\
\hline Linear NARMAX & 20.92 \\
\hline Quadratic NARMAX & $\mathbf{1 8 . 3 1}$ \\
\hline Linear-Nonlinear model & 32.29 \\
\hline
\end{tabular}

\section{CONCLUSIONS}

Modelling biological systems is difficult due to insufficient knowledge about the internal components and organisation, and the complexity of the interactions within the system. Existing computational models of visual neurons can be derived by quantitatively fitting particular sets of physiological data using an input-output analysis where a known input is given to the system and its output is recorded as illustrated in the LinearNonlinear approach in Section 4.4.

At a computational level we have presented the use of the NARMAX system identification technique to accurately model individual retinal ganglion cells as shown in Section 4.2 and Section 4.3. We have presented a comparison of the actual neuronal response and provided a comparison with the actual neuronal response. Visual comparison illustrates that all the methods can model the neuronal response with accuracy although some errors are present. Computing the RMSE provides a more quantitative measure of error and the results summarised in Table 1 illustrate that the quadratic NARMAX model performs substantially better than 
both the LNL model and the linear NARMAX model.

Using NARMAX system identification techniques to express the biological input-output coupling mathematically we have modelled highly complex neuronal structures, and thus "identified" ganglion cell behaviour with visual scenes. These polynomial models represent the mapping between perception and response. The next stage in this work will be to increase the complexity of the stimulus by having spatially varying stimuli; we have already started to test the effectiveness of this using the natural image sequences.

\section{ACKNOWLEDGEMENTS}

The research leading to these results has received funding from the European Union Seventh Framework Programme (FP7-ICT-2011.9.11) under grant number [600954] ("VISUALISE"). The experimental data contributing to this study have been supplied by the "Sensory Processing in the Retina" research group at the Department of Ophthalmology, University of Göttingen as part of the VISUALISE project.

\section{REFERENCES}

Herikstad, R., Baker, J., Lachaux, J.-P., Gray, C. M., \& Yen, S.-C. (2011). Natural Movies Evoke Spike Trains with Low Spike Time Variability in Cat Primary Visual Cortex. Journal of Neuroscience, 31(44), 15844-15860. doi:10.1523/JNEUROSCI.5153-10. 2011

De Boer, Kuyper, P. (1968). "Triggered Correlation". Biomedical ngineering, vol.BME-15, no.3, pp.169179. doi: 10.1109/TBME.1968.4502561 Transactions

Sakai,H.M., Naka K.I., Korenberg, M.J. (1988) "Whitenoise analysis in visual neuroscience". Visual Neuroscience, 1, pp 287-296 DOI: 10.1017

Chichilnisky EJ (2001) A simple white noise analysis of neuronal light responses. Network 12(2):199-213.

Talebi, V., Baker, C.L. (2012). "Natural versus Synthetic Stimuli for Estimating Receptive Field Models: A Comparison of Predictive Robustness". The Journal of Neuroscience, Vol. 32, No. 5., pp. 1560-1576, doi: 10.1523

Marmarelis, P.Z., Naka, K.I. (1972). White-noise analysis of a neuron chain: An application of the wiener theory. Science 175, 1276-1278

Victor, J., Shapley, R., Knight, B. (1977). Nonlinear analysis of cat retinal ganglion cells in the frequency domain. Proc. Natl. Acad. Sci. U.S.A. 74(7), 30683072
Victor, J. (1979) Nonlinear systems analysis: comparison of white noise and sum of sinusoids in a biological system. Proc. Natl. Acad. Sci. U.S.A. 76(2), 996-998

Marmarelis, V. (2004) Nonlinear Dynamic Modeling of Physiological Systems. Wiley Interscience, Hoboken.

Korenberg, M., Hunter, I. (1996). The identification of nonlinear biological systems: Volterra kernel approaches. Ann. Biomed. Eng. 24(2), 250-268.

Marmarelis VZ, Zhao X. (1997). Volterra models and three-layer perceptions. IEEE Trans Neural Networks $8: 1421$.

Block-oriented Nonlinear System Identification (2010), Lecture Notes in Control and Information Sciences, Springer Berlin / Heidelberg, Vol. 404. Giri, F. and Bai E.W. Eds

Nehmzow, U. (2006) Scientific Methods in Mobile Robotics: quantitative analysis of agent behaviour. Springer, 2006.

Ostojic S, Brunel N (2011) From Spiking Neuron Models to Linear-Nonlinear Models. PLoS Comput Biol 7(1): e1001056. doi:10.1371/journal.pcbi.1001056

Pillow JW, Paninski L, Uzzell VJ, Simoncelli EP, Chichilnisky EJ. (2005). Prediction and decoding of retinal ganglion cell responses with a probabilistic spiking model. JNeurosci.23;25(47):11003-13.

Korenberg MJ. (1991). Parallel cascade identification and kernel estimation for nonlinear systems. Ann Biomed Eng 19:429.

Pillow JW, Shlens J, Paninski L, Sher A, Litke AM, Chichilnisky EJ, Simoncelli EP. (2008) Spatio temporal correlations and visual signaling in a complete neuronal population. Nature 454: 995-999

Billings SA, Voon WSF. 1984. Least-squares parameter estimation algorithms for non-linear systems. Int.J Systems Sci 15:601.

Friederich, U., Coca, D., Billings, S.A., Juusola, M. (2009) Data Modelling for Analysis of Adaptive Changes in Fly Photoreceptors. Proceedings of the 16th International Conference on Neural Information Processing: Part I

Z. Song, S.A. Billings, D. Coca, M. Postma, R.C. Hardie, \& M. Juusola. (2009), Biophysical Modeling of a Drosophila Photoreceptor. LNCS (ICONIP 2009, Part I) $5863: 57-71$.

Kerr, D, Nehmzow, U and Billings, S.A. (2010) Towards Automated Code Generation for Autonomous Mobile Robots. In: The Third Conference on Artificial General Intelligence, Lugano. Switzerland. Atlantis Press, Scientific Publishing, Paris, France. 5 pp.

Billings, S.A. Nonlinear system identification: NARMAX methods in the time, frequency, and spatio-temporal domains. John Wiley \& Sons, 2013.

Lau B, Stanley GB, Dan Y (2002) Computational subunits of visual cortical neurons revealed by artificial neural networks. Proc Natl Acad Sci U S A 99: 8974-8979.

Prenger, R., Wu, M.C.K., David, S.V., Gallant, J.L., (2004) Nonlinear V1 responses to natural scenes revealed by neural network analysis, Neural Networks, Volume 17, Issues 5-6, Pages 663-679, 10.1016/ j.neunet.2004.03.008. 